EPJ Web of Conferences 87,02010 (2015)

DOI: $10.1051 /$ epjconf/20158702010

(C) Owned by the authors, published by EDP Sciences, 2015

\title{
Machine safety issues with respect to the extension of ECRH systems at ASDEX Upgrade
}

Martin Schubert ${ }^{\mathrm{a}}$, Albrecht Herrmann, Francesco Monaco, Volker Rohde, Harald Schütz, Jörg Stober, Thomas Vierle, Stefan Vorbrugg, Dietmar Wagner, Dieter Zasche, Thomas Zehetbauer, Wolfgang Zeidner and ASDEX Upgrade Team

Max Planck Institute for Plasma Physics, Boltzmannstr. 2, 85748 Garching, Germany

\begin{abstract}
The beam intensity of electron cyclotron resonance heating at ASDEX Upgrade has the potential to seriously damage in-vessel components, whenever not fully absorbed by the plasma. Operation is, therefore, interlocked with both plasma current and density above a given threshold. Microwave protection detectors installed in several ports on the low field side switch the heating system off, in case the stray radiation exceeds a given threshold. During regular inspections, however, damages were reported in the vicinity of the launchers and in particular around the tiles of the heat shield. On one hand, it was found that insulating material, which may not face the plasma, degraded due to millimetre wave absorption. The waves entered the free space behind the heat shield through gaps. On the other hand, local damage even of metallic components was observed on surfaces, which were directly exposed to the microwave beam. Polarisation errors, which led to a local shine through of significant beam power, were responsible. We note that this happened mainly on the high field side in a certain distance to the microwave protection detectors, which were not triggered by the events. In order to increase the level of protection, we identify three necessary measures: Firstly, polarisation control is to be automated such, that mode content and shine through can be monitored. Secondly, by installing additional detectors, the spatial coverage of stray radiation monitoring is enlarged. Thirdly, the heat shield tiles will be redesigned in order to increase the shielding against millimetre waves.
\end{abstract}

\section{Introduction}

Electron cyclotron resonance heating $(\mathrm{ECRH})$ power at ASDEX Upgrade (AUG) will be increased [1] and its operational space is being extended [2]. When using O-2 or X-3 mode, the optical thickness of typical AUG plasmas can be moderate (Figures 1 and 2). Incomplete absorption gives rise to a shine through on $\mathrm{AUG}^{\prime} \mathrm{s}$ heat shield (HS). We would like to point out, that even in the most commonly used X-2 scheme with a nominal absorption beyond $99 \%$ [3], a polarisation error causes a shine through, since the cross polarisation is indeed $\mathrm{O}-2$.

We briefly review system components in sections 2-4 and report on damages in section 5. The experimental results in section 6 indicate that the most severe damages occur, whenever there is a shine through of the primary beam, causing locally a high power density. Based on this analysis, we deduce the necessary measures.

\section{AUG ECRH setup}

A detailed description of the ECRH system can be found elsewhere [4]. Here, we point out some issues relevant for machine safety. Since polarisation plays a crucial role, this is also briefly reviewed.

\subsection{Hazard potential}

The ECRH beams at AUG are designed using Gaussian optics with a beam waist $w_{0}=1.7 \mathrm{~cm}$ inside the plasma, about $47.5 \mathrm{~cm}$ after the launcher mirror. At this position the most powerful beamline (ECRH \#7 / $900 \mathrm{~kW})$ generates a microwave intensity of $200 \mathrm{~kW} / \mathrm{cm}^{2}$. After the beam waist, diffraction causes widening. On the HS the beam radius $w=3.6 \mathrm{~cm}$ corresponds to a maximum of $45 \mathrm{~kW} / \mathrm{cm}^{2}$ in the absence of beam absorption. This value scales with the actual shine through fraction in the case of plasma heating. The data correspond to $140 \mathrm{GHz}$. Intensities are smaller for any other operating frequency.

The majority of AUG experiments uses X-2 heating (Figure 1), where plasma absorption reaches values of $99 \%$ [3]. ECRH operation is, therefore, interlocked with plasma current and density above a given threshold. The plasma density, however, may dynamically increase up to the X-mode cut-off $\left(n_{\mathrm{e}}=1.2 \cdot 10^{20} \mathrm{~m}^{-3}\right.$ at $\left.B=2.5 \mathrm{~T}\right)$. In that case the wave is reflected and ends up as stray

\footnotetext{
a Corresponding author: Martin.Schubert@ipp.mpg.de
} 


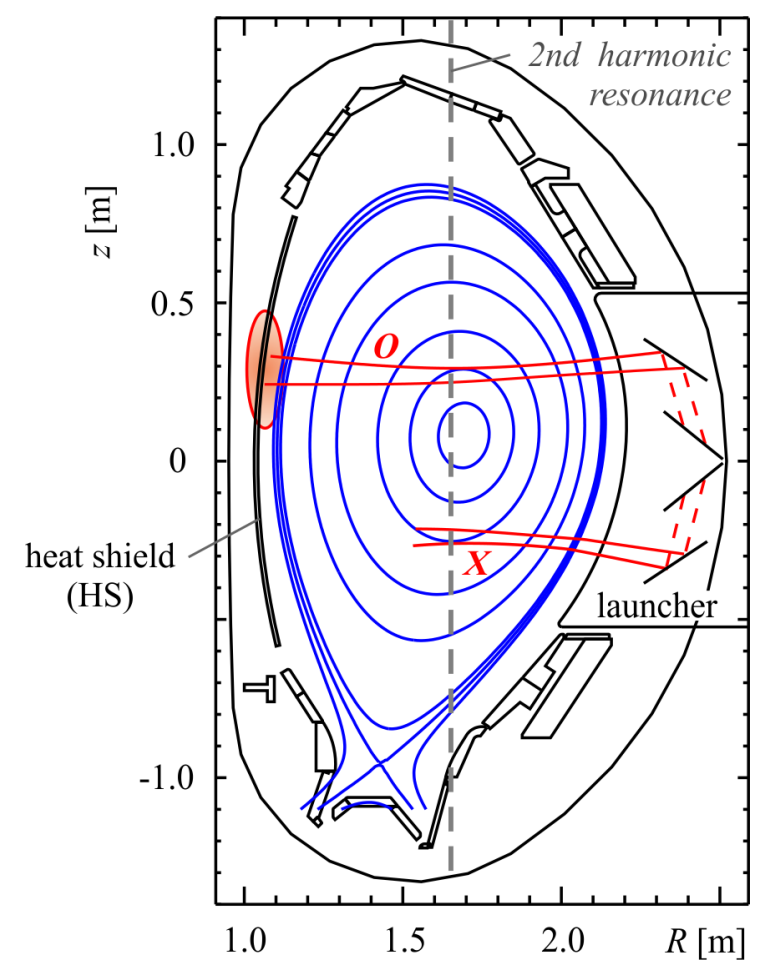

Figure 1. Schematic AUG cross section with two ECRH launchers on the low field side. Mainly the $2^{\text {nd }}$ harmonic is used. An O-mode content due to incorrect beam polarisation will have a shine through on the heat shield (HS), as indicated for the upper beam. For a pure X-mode polarisation the optical thickness can reach values of $\tau=100$ [3]. In that case $>99 \%$ of the beam power is absorbed by the plasma.

radiation on the low field side. Microwave detectors installed in several ports (see section 4) switch the ECRH off, in case a given threshold is exceeded.

It should be mentioned that plasma fluctuations and turbulence generate stray radiation by beam scattering. The scattering cross section depends on the ratio between the local density and the cut-off density [5]. At AUG, part of the background stray radiation (section 6) is due to this effect, but it is small compared to polarization issues. In order to make predictions for future machines and heating schemes, theoretical studies and simulations are ongoing, e.g. [6].

\subsection{Polarisation control}

Beam polarisation at the Gyrotron output window is linear. A pure wave mode in the plasma requires an elliptical polarisation at the vacuum plasma interface (Figure 3), depending on the angle between the beam vector $\boldsymbol{k}$ and the magnetic field vector $\boldsymbol{B}$. Two polarisers, rotatable grooved mirrors, are used to adjust the polarisation in each beamline.

In the multi-frequency ECRH system at AUG, the polarisation of the launched wave can be modelled [7] as a function of the frequency and of the two polariser angles. Directional couplers, sensitive to one component of the wave electric field, are installed in the last mitre bend (Figure 4), close to the launcher. The consistency of the polarisation can be checked, if the appropriate component of the model electric field is compared to the

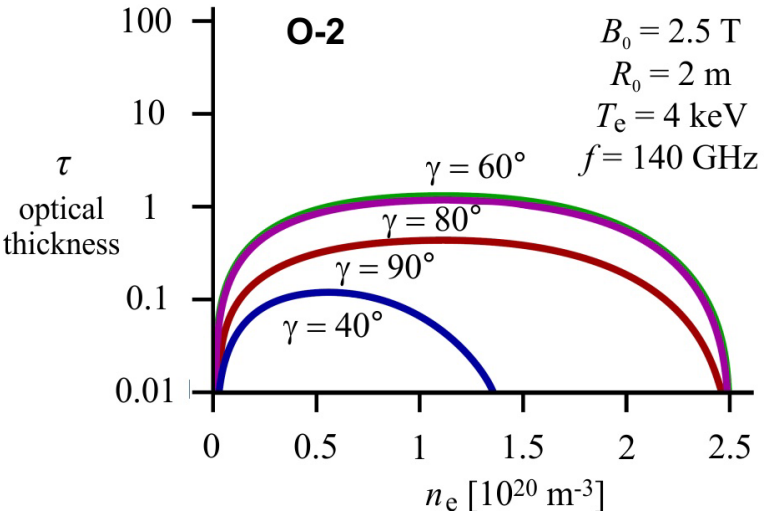

Figure 2. Optical thickness $\tau$ of the O-2 mode [3] as a function of the plasma electron density $n_{\mathrm{e}}$ and launch angle $\gamma$. The shine through fraction after the first pass is $\propto \exp (-\tau)$.
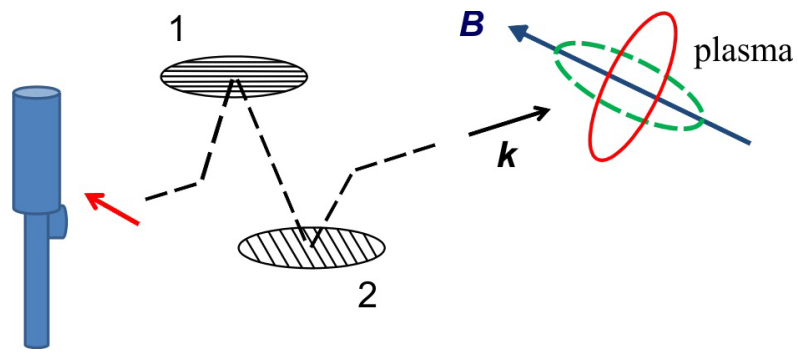

Figure 3. Schematic polarisation system in an ECRH beamline. Two rotatable polarisers $\mathbf{1}$ and $\mathbf{2}$ allow to match any elliptical polarisation, defined by the magnetic field vector $\boldsymbol{B}$, the beam vector $\boldsymbol{k}$ and the mode. Aligned in direction of $\boldsymbol{B}$ (dashed ellipse) is the ordinary $(\mathrm{O})$ mode, while the (solid) ellipse $\perp \boldsymbol{B}$ corresponds to the extraordinary (X) mode. The angle between $\boldsymbol{k}$ and $\boldsymbol{B}$ determines the ellipticity and $\boldsymbol{k} \perp \boldsymbol{B}$ corresponds to a linear polarisation.

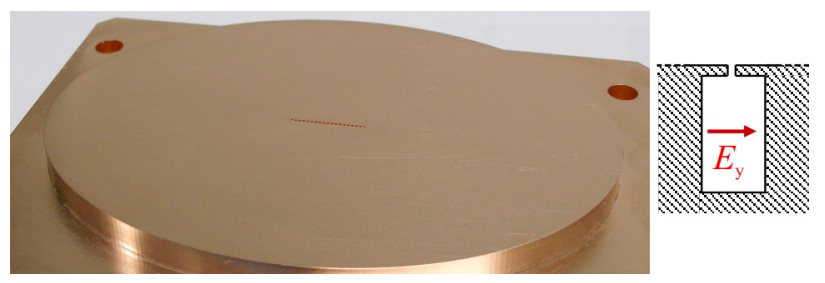

Figure 4. Mitre bend mirror surface (left) with built-in directional coupler (coupling holes in the center). Only one component of the electric field can generate a propagating mode in the pick-up waveguide below the mirror surface.

measurement as a function of the two polariser angels (Figure 5) for each frequency. This is done at least once for each new beamline or Gyrotron.

For plasma heating the nominal polarisation is calculated from the planned $\boldsymbol{k}$ and $\boldsymbol{B}$ vectors, where $\boldsymbol{k}$ is defined by a vertical and a toroidal angle and $\boldsymbol{B}$ by the toroidal field strength and the nominal plasma current. These four input values are communicated to the ECRH operator by the AUG experiment leader. For the corresponding polarisation, a 2D map similar to Figure 5 is generated and a polariser setting (i.e. two polariser angles) with mode purity $>99 \%$ is selected. When all settings are complete, the AUG experiment countdown is initiated. Routinely no further independent consistency checks are performed. 


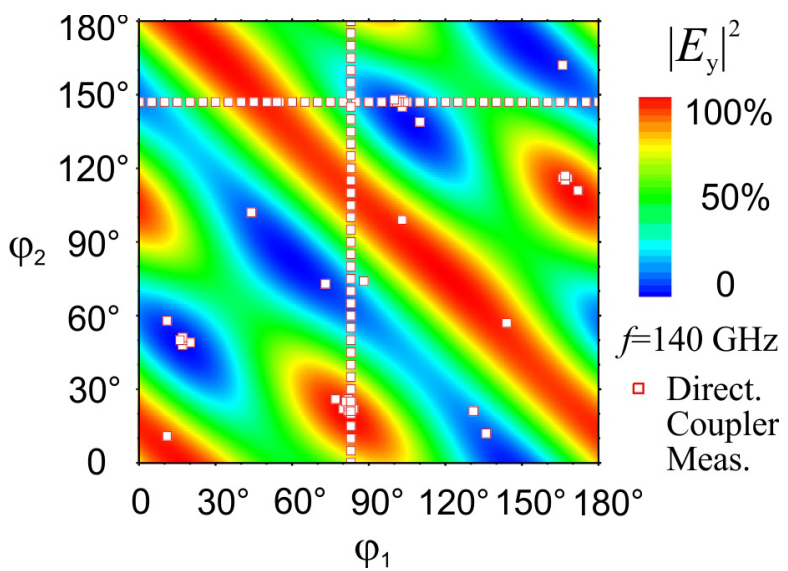

Figure 5. ECRH \#6: Square of the normalized electric field component perpendicular to $\boldsymbol{k}$ and parallel to the mitre bend mirror surface, function of the two polariser angles. The model value (color map) for a given input power can be compared to the directional coupler measurement (small squares). Measurement values are not shown. More details in [7].

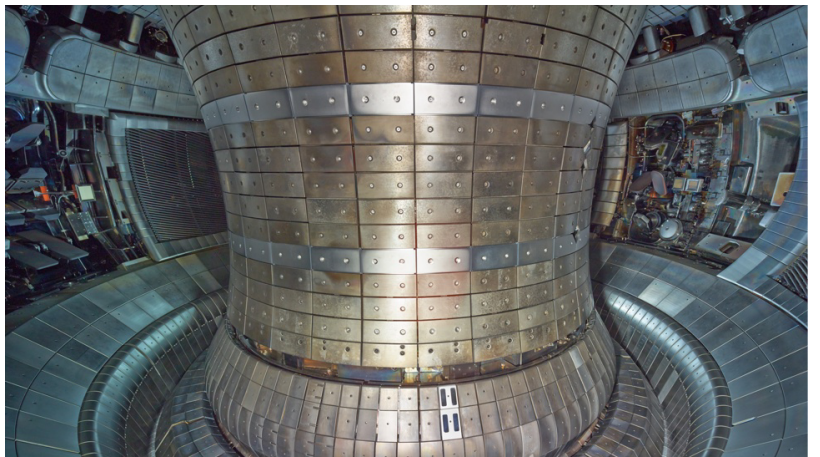

Figure 6. The heat shield (HS): tungsten coated graphite tiles cover the central column. Two horizontal rings (slightly brighter) are made of $\mathrm{P} 92$ steel for material testing. There is a nominal gap of four millimetres between the tiles.

\section{Heat shield}

AUG's heat shield was originally designed to withstand the heat flow out of the scrape-off layer plasma at this position. As it is shown in Figures 6 and 7, the HS consists of tiles, mounted on an actively cooled rectangular cooling pipe. In the original design there is a nominal gap of $4 \mathrm{~mm}$ around each tile, which allows the $140 \mathrm{GHz}$ millimetre wave to pass through. Electric installations in the free space behind the tiles have suffered damage (see section 5).

In 2013 during vacuum vessel maintenance several radiation protective measures were installed in sectors where high power millimetre waves are launched. Metal strips coated with a ceramic $\mathrm{Al}_{2} \mathrm{O}_{3} / \mathrm{TiO}_{2}$ layer, as well as PEEK foil strips were attached to the fittings, bridging the gap between the tiles (Figure 8) behind the HS. The ceramic coating significantly absorbs millimetre waves, while the PEEK strips are used as markers. PEEK will change its colour and finally carbonize, when heated up by millimetre wave absorption. The used PEEK foil has a thickness of $d=0.125 \mathrm{~mm}$, density $\rho=1.3 \mathrm{~g} \mathrm{~cm}^{-3}$ and a specific heat $c=0.32 \mathrm{~J} \mathrm{~g}^{-1} \mathrm{~K}^{-1}$. Temperature stability is specified until $300{ }^{\circ} \mathrm{C}$.

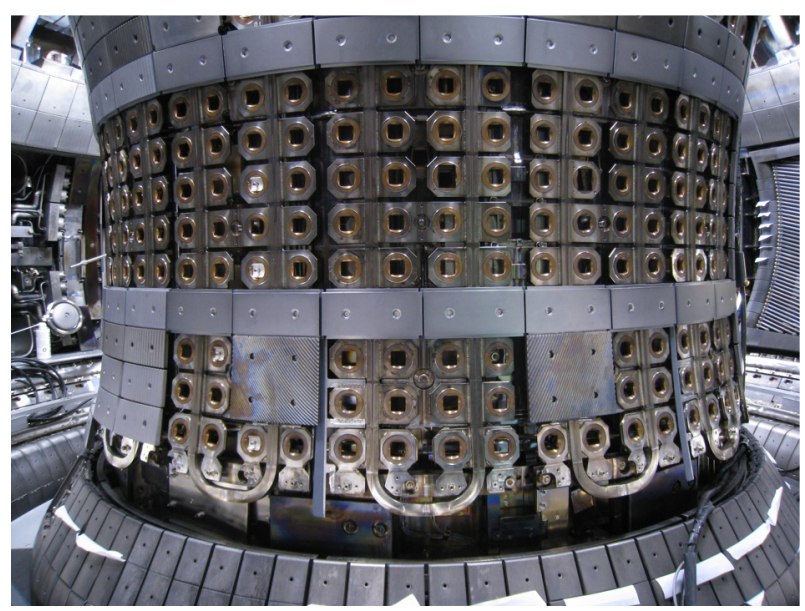

Figure 7. Partly disassembled heat shield. Ear-shaped fittings for the tiles are attached to vertical (U-shape) cooling pipes. Free space behind the fittings is used for electric installations. The two rings of P92 steel tiles are mounted. The edges of these tiles have a newly designed overlapping structure.

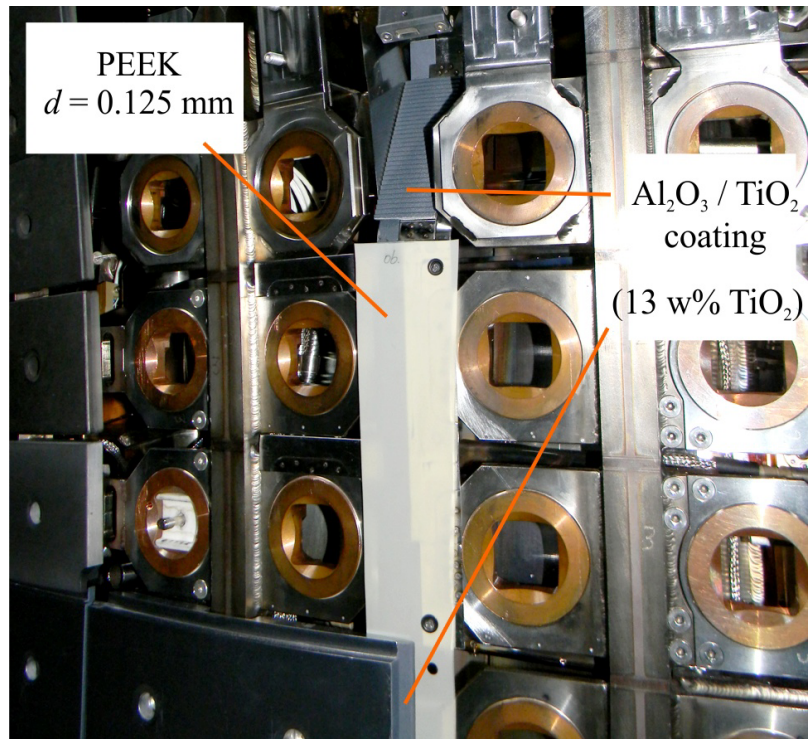

Figure 8. Test of millimetre wave protection: ceramic coating absorbs wave power near the opening of a sensitive pinhole camera and in the overlapping structure of the P92 steel tiles. PEEK strips serve as markers for absorbed power.

\section{Stray radiation detection}

Several millimetre wave detectors, installed in ports on the low field side (Figure 9) are distributed around the torus (Figure 10). An interlock circuit switches the ECRH off, if the detected (unabsorbed) power is above a given threshold. Different detector types have been investigated [8]. We note that SP5, SP9 and SP14 are Sniffer probes [9], while D4 and D6 are D-band pick-up waveguides, mounted onto a quartz vacuum window. Typical detector signals will be shown in section 6 .

In 2012 severe damage from non-absorbed millimetre waves occurred in vessel sector 4 (see section 5). Signal levels in the Sniffer probes SP5, SP9 and SP14 were below the threshold during this accident. We assume that due to the specific beam geometry, a local stray radiation peak existed in sector 4 . With the two new measurement 
channels D4 and D6 we enlarge our monitored region in order to prevent similar damage in the future.

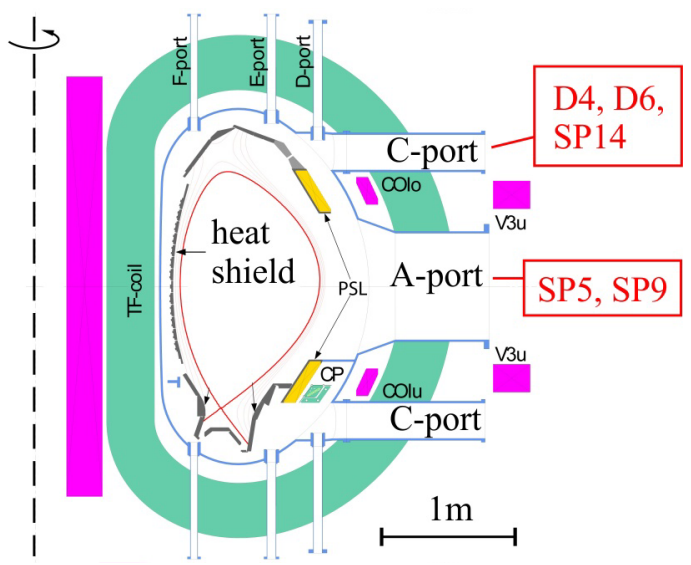

Figure 9. Schematic cross section with ports on the low field side of AUG. The positions of millimetre wave stray radiation detectors are indicated. 'SP' denotes a Sniffer sphere and 'D' a simple D-band pick-up waveguide with a beam-lead diode, mounted behind a quartz vacuum window. The number indicates the vessel sector (see also Figure 10).

\section{Reported damage}

During plasma experiments, situations occurred with a transient and local ECRH shine through on the heat shield. In this case the electric field strength was large enough to ignite an arc, visible in the video recording (Figure 11). The arc is characterised by a small and bright spot on the heat shield in an area which is dark under normal conditions.

During regular maintenance phases, the vacuum vessel interior is inspected carefully in order to identify and repair damages. Since millimetre waves can penetrate through the gaps between tiles, the heat shield is partly disassembled. Figure 12 shows a set of typical minor damages. Insulating material that significantly absorbs millimetre waves is concerned in particular. Most of these issues could be repaired easily, given the required maintenance access.

In 2012 a more serious damage occurred during experiment \#28017. Figure 13 shows the location in sector 4, the open space below the heat shield. Here, a magnetic pick-up coil was mounted behind a thin stainless steel cover. Due to an accidental O-mode polarisation and a specific beam geometry with co-ECCD close to the plasma edge, the beam shine through directly hit the cover, damaged it and also destroyed the coil inside the casing (see also Figure 13). The power on-time of ECRH was 2.5 seconds.

The signals of the stray radiation monitors were investigated. At that time, only SP5, SP9 and SP14 were in operation (see Figure 10). Notably, the signal of SP5 was far below the interlock level, although the used launcher ECRH \#7 is located in the same port as SP5. Due to the large toroidal angle, the beam shine through and stray radiation ended up in sectors 4 and 3, far enough from sector 5 . We note also that the signal of SP14 was clearly elevated, but still below the interlock

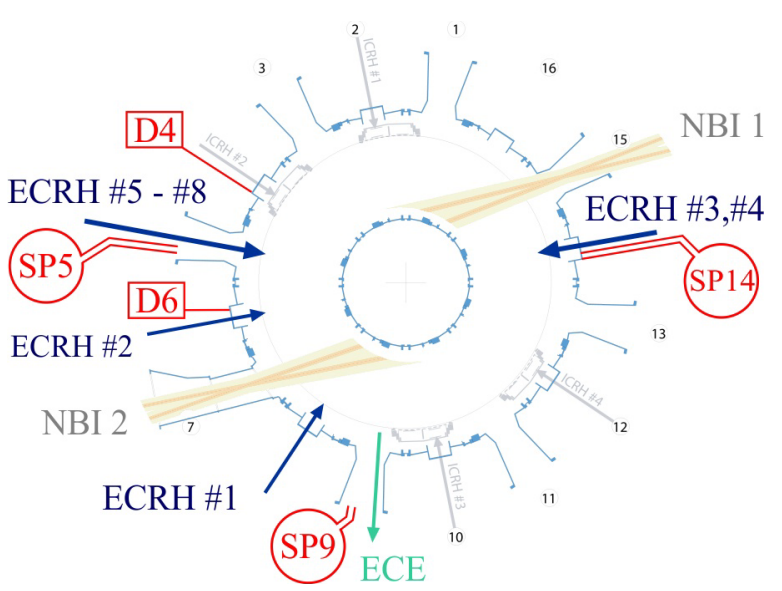

Figure 10. Top View of an equatorial cross section through the AUG vacuum vessel, indicating the position of heating systems and millimetre wave detectors ( $\mathrm{SP} n, \mathrm{D} n)$. ECE is the electron cyclotron emission measurement system, which includes also a power measurement of the stray radiation. Detector positions are close to the ECRH launchers. 'D4' and 'D6' have been installed recently in order to enlarge the monitored region around the ECRH launchers \#5 - \#8.

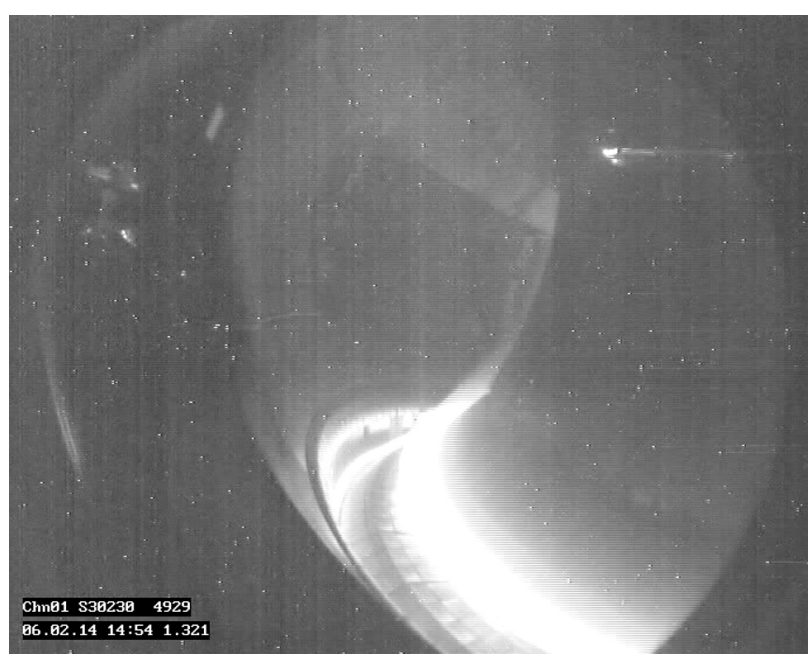

Figure 11. Video snapshot from AUG experiment \#30230. In the upper right corner an arc can be seen on the HS. This was ignited by an ECRH beam shine through. Under normal conditions this area is dark. Most of the light comes from the divertor region at the bottom of the machine.

level. We conclude that the distance between ECRH \#7 and SP14 was too large for a reliable stray radiation detection and, therefore, initiated the installation of the new D4 detector [10].

The investigation revealed also that the previous discharge \#28016 was a ctr-ECCD experiment with the same absolute value but opposite sign of the toroidal beam angle. From \#28016 to \#28017 the toroidal beam angle had been flipped, but not the polarisation. All necessary modifications including the new polarisation were correctly written in the lab journal, but the settings were, however, not realized. Most likely the operator in charge had been disturbed while entering the new parameters into the control system. Switching the toroidal angle without changing the polarisers resulted in an almost pure O-mode. 


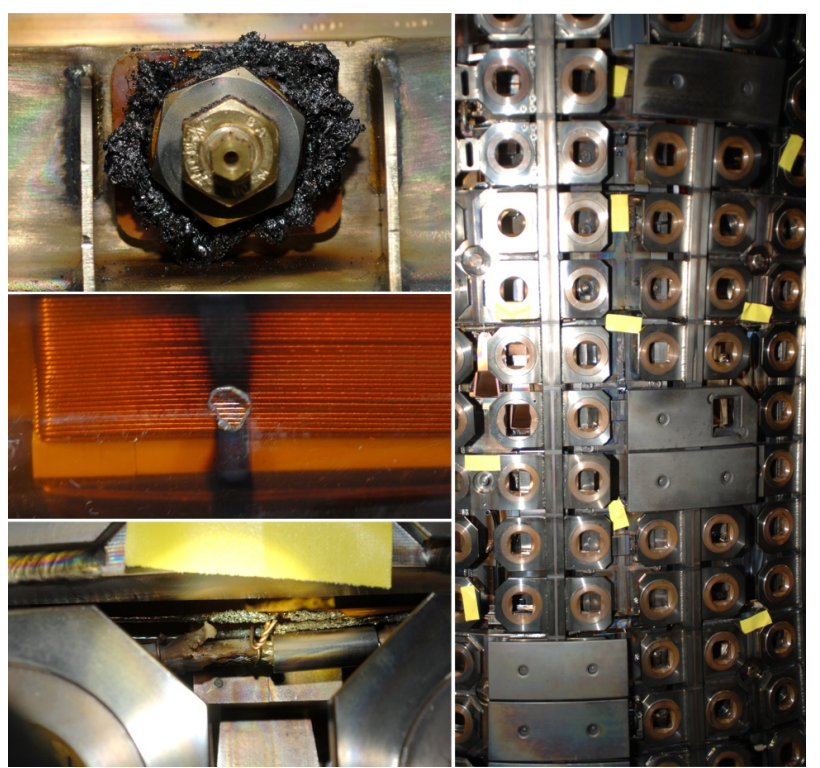

Figure 12. Left: various ECRH related damage. Insulating material that absorbs millimetre waves can be carbonized and evaporated, as it is shown in the two upper photos on the left. Even the shadow of the gap between tiles is visible as a vertical black stripe on the Kapton foil (second photo). A damaged cable is shown in the lower photo. Right: Part of the disassembled heat shield with yellow tape markers for any damage that was found during inspection.

Since human errors cannot be excluded, we conclude that polarisation should be automatically controlled. This requires, however, extended input for the control system, including the planned beam geometry $\boldsymbol{k}$, the planned magnetic field vector $\boldsymbol{B}$ and the planned mode of the ECRH beam. The automated module then should check whether the polarisation defined by $\boldsymbol{k}$ and $\boldsymbol{B}$ is consistent with the planned mode.

\section{Experimental results}

As another example we show the result of a stray radiation measurement in AUG discharge \#30587. All four launchers (ECRH \#5-\#8, Figure 10) in sector 5 were in operation. They injected up to $2.8 \mathrm{MW}$ in X-2 mode (Figure 14). The beam vectors $\boldsymbol{k}$ were perpendicular to the magnetic field vector $\boldsymbol{B}$. The stray radiation intensity is highest in the launching port itself and reaches values up to $6 \mathrm{~W} / \mathrm{cm}^{2}$ (Figure 15). This includes diffraction losses from the finite-sized focussing and launching mirrors at this end of the transmission line. In any adjacent sector, the intensity is less than $-10 \mathrm{~dB}$ (Figure 15). This indicates that even the diffuse and scattered stray radiation can be confined within one sector, and it corroborates the need of a sufficient number of measurement channels in different sectors.

Figure 15 shows also that the measurement results from the calibrated Sniffer probe SP9 are essentially the same as those from the pick-up D-band waveguide D4. The neighbouring D6 yielded similar results. Both D4 and D6 were calibrated, using the power measured by the detector diode, divided by the waveguide cross section. With respect to this simple operation, the calibration of the Sniffer probe needed much more elaboration [11].

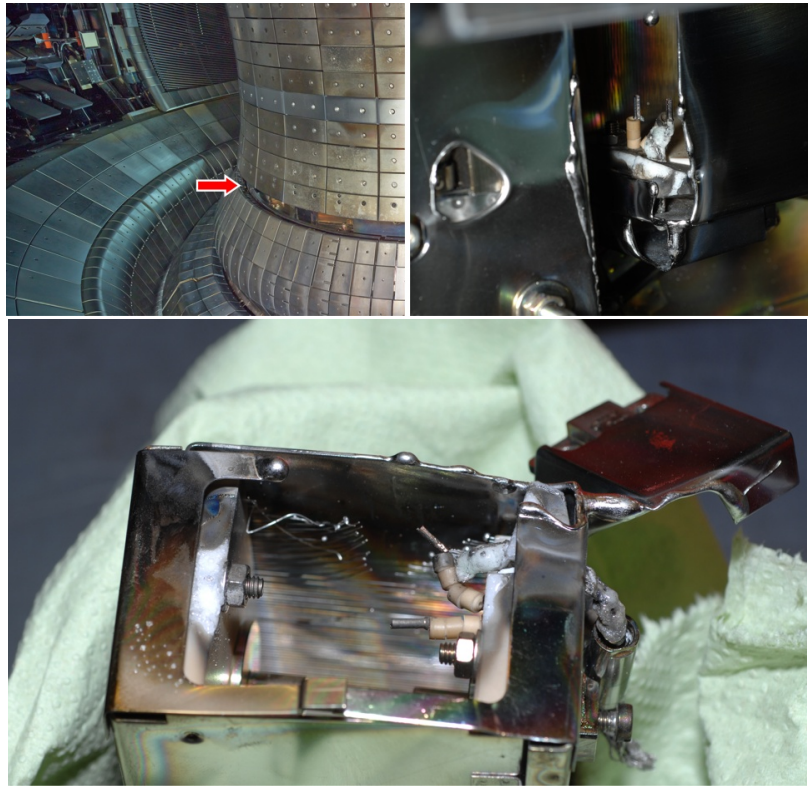

Figure 13. In 2012 a pick-up coil was destroyed by a $2.5 \mathrm{sec}$ ECCD pulse, which had an accidental O-Mode polarisation and, thus, a shine through at the indicated position.

Traces of ECRH shine through on the PEEK strips of the HS (Figure 8) could be evaluated in February 2014. One of the polarisers of ECRH \#8 had an angular offset, probably due to an error in the polariser rotation controller. A 5 sec pulse (\#30227) with an estimated 30\% O-Mode content generated visible arcing on the HS. The problem was identified in the subsequent discharges, where only short pulses of ECRH \#8 were applied. On the next day, the AUG vessel was vented with air and the concerned PEEK was replaced. Figure 16 shows the strip that was taken out.

We made a calorimetric estimate of the millimetre wave power absorbed by the PEEK foil. The uncertainty is large, since neither the time scale on which the damage evolved, nor the dielectric properties of PEEK at 140 $\mathrm{GHz}$ are known precisely. Based on the delay between ECRH power and the arcing that was visible in the video observation, we assumed that the foil was heated from ambient temperature to its destruction limit $\left(343{ }^{\circ} \mathrm{C}\right)$ within roughly $100 \mathrm{~ms}$. The heat capacitance per area is calculated from the material properties (section 3) and amounts $0.0052 \mathrm{~J} \mathrm{~K}^{-1} \mathrm{~cm}^{-2}$. Hence, we deduce the absorbed power per area of $17 \mathrm{~W} \mathrm{~cm}^{-2}$.

If we assume $\varepsilon^{\prime}=3$ and $\tan \delta=0.01$, based on dielectric data at lower frequencies, then this foil absorbs $1.3 \%$ of the millimetre wave power. Accordingly, the shine

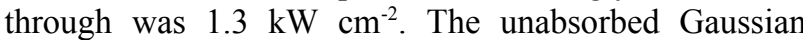
beam of ECRH \#8 would have had a maximum beam intensity of $30 \mathrm{~kW} \mathrm{~cm}^{-2}$ at this position.

\section{Discussion}

Although calorimetric analysis of the traces on the PEEK strip has large uncertainties, the overall picture is consistent, if we assume that the polarisation error is responsible for the observations. Comparing the intensities involved, there are nearly three orders of 


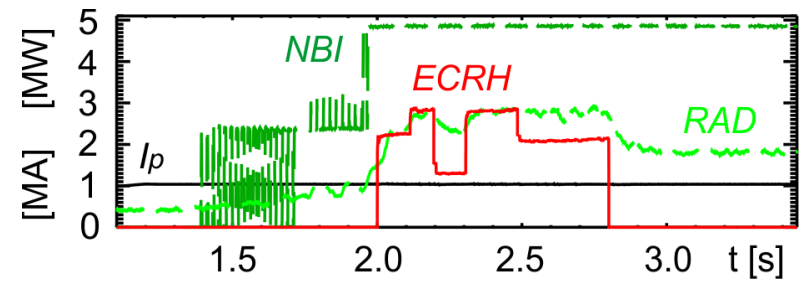

Figure 14. AUG \#30587: injected ECRH and NBI power. Radiation loss and flat-top plasma current Ip are overplotted.

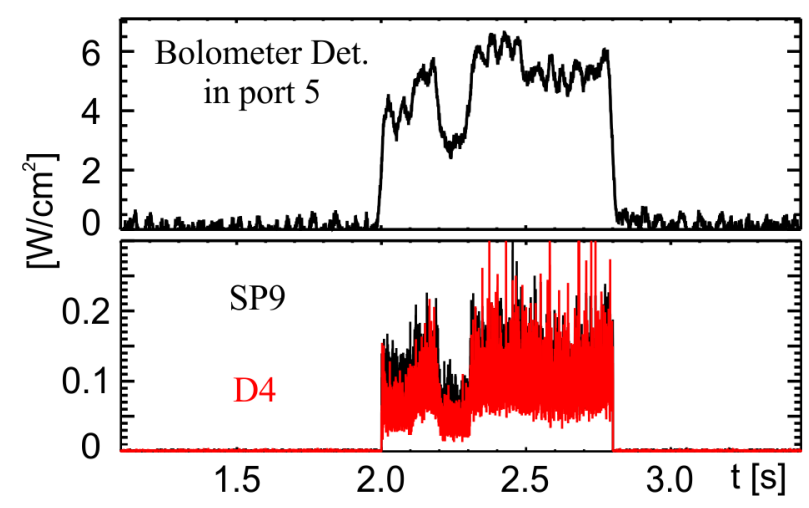

Figure 15. AUG \#30587: measured ECRH stray radiation. Top: Calibrated Bolometer detector $[8,11]$ installed near SP5. Bottom: Calibrated measurements with $0.2 \mathrm{~ms}$ sliding average from SP9 (black) and D4 (red).

magnitude between an O-Mode shine through $\left(\mathrm{kW} \mathrm{cm}^{-2}\right)$ and the stray radiation in the launcher port $\left(\mathrm{W} \mathrm{cm}^{-2}\right)$. At AUG with its maximum pulse length of $10 \mathrm{sec}$, the hazard potential of stray radiation (from beam diffraction and scattering) will always be small compared to transient events with shine through or reflection of an ECRH beam. Emphasis should therefore be put on an independent control scheme for the polarisation state and the potential shine through.

The Sniffer probes and other millimetre wave detectors are very important to detect reflection events, e.g. due to a rise in plasma electron density that generates strong refraction or cut-off. Since this can happen locally and with a compact beam, it is essential to have good coverage of the plasma surface by a sufficient number of detection channels. Multiple reflections will occur in such case, which relaxes the requirement a little bit. The topology of the vacuum vessel also plays an important role. At AUG we think that one detector per vessel sector is sufficient in the vicinity of the launchers, at least in order to actively protect the low field side. On the high field side, space restrictions presently do not allow such installations.

A pragmatic approach to protect the heat shield is the redesign of the tiles. Compared to the original design with open gaps, the situation improved, where the new overlapping tiles with an absorbing coating were installed. Presently, it is evaluated, whether the components can withstand the worst case scenario with an unabsorbed beam. These efforts include laboratory measurements of the shielding at $140 \mathrm{GHz}$ by the labyrinth-like structure in the overlap.
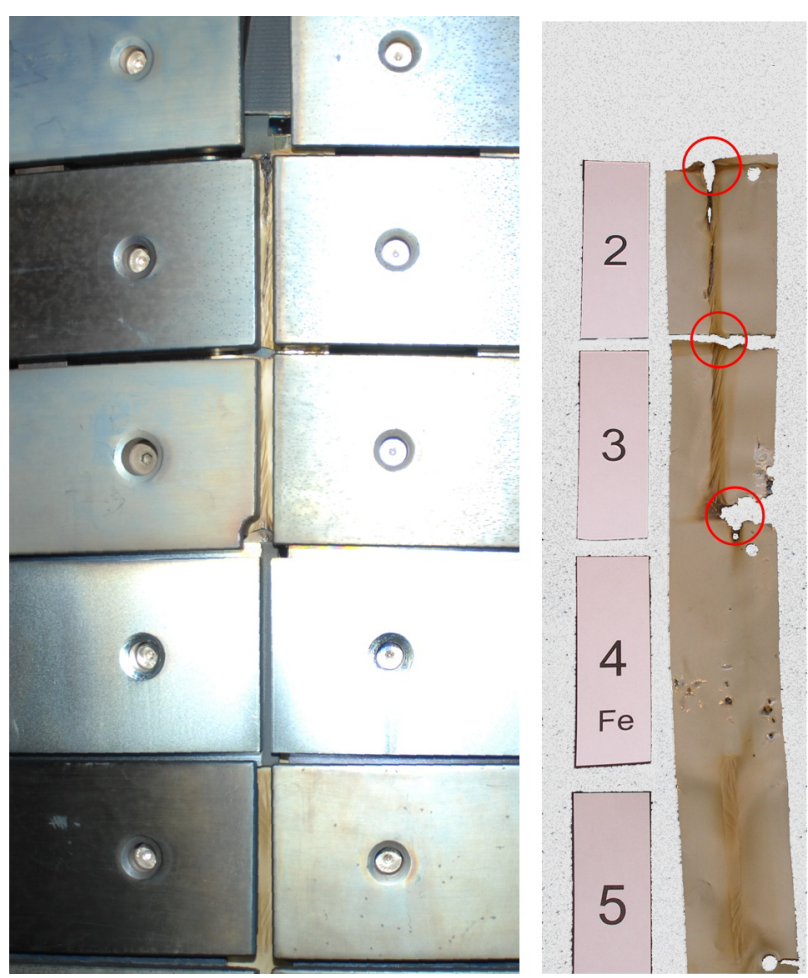

Figure 16. Traces of absorbed ECRH power on PEEK, which was mounted behind the gap. The uncovered parts clearly changed colour. In rows 2 and 3, at intersections of vertical and horizontal gaps, it was completely burned through. According to the video analysis, this corresponds to where the ECRH shine through hit the tiles. Such traces do not exist in row 4, where the overlapping Fe tiles were mounted.

\section{Acknowledgement}

This project has received funding from the European Union's Horizon 2020 research and innovation programme under grant agreement number 633053. The views and opinions expressed herein do not necessarily reflect those of the European Commission.

\section{References}

1. J. Stober et al., EPJ Web Conf 32, 02011 (2012)

2. H. Höhnle et al., Nucl. Fusion 51, 083013 (2011)

3. V. Erckmann, U. Gasparino, Plasma Phys. Control. Fusion 36, 1869 (1994)

4. D. Wagner et al., J Infr Milli Tera 32, 274 (2011)

5. M. Schubert et al., EDP Sciences NAMES 2007, http://dx.doi.org/10.1051/names2007026 (2008)

6. E. V. Sysoeva et al., Plasma Phys. Control. Fusion 55, 115001 (2013)

7. D. Wagner et al., Int J Infrared Milli 26, 163 (2005)

8. M. Schubert et al., EPJ Web Conf 32, 02013 (2012)

9. F. Gandini et al., Fusion Eng Des 56-57, 975 (2001)

10. D. Schmid-Lorch, dipl. thesis, Univ. Ulm, IPP report 1/345, http://edoc.mpg.de ID=579778 (2012)

11. F. Honecker, dipl. thesis, TU München, IPP report 1/348, http://edoc.mpg.de ID=669361 (2013) 